\title{
En conversant avec les bourreaux
}

\author{
Déni et valeurs morales exprimées en situation \\ d'entretien (Allemagne nazie ; dictatures du Plan \\ Condor)
}

\author{
Par Ilan Lew \\ (Doctorant en sociologie au CADIS-EHESS)
}

\section{Résumé/Abstract}

[Fr] Dans cet article, nous mettons en perspective des témoignages d'auteurs de violences de masse ayant sévi dans des contextes socio-historiques bien distincts, mais qui ont cela en commun qu'ils proviennent tous de situations extra-judiciaires, souvent carcérales, et prennent la forme de conversations avec des journalistes ou des codétenus. En premier lieu, nous donnerons à voir les enjeux de pouvoir qui caractérisent de telles situations d'interaction, ainsi que les stratégies déployées pour faire sortir ces "vétérans" de leur discours de façade et les faire revenir sur leurs violences. Dans un second temps, nous nous penchons sur ce qui, dans des documents de ce type, pourrait nous donner un accès privilégié à la question de la morale dans la violence de masse. À cette fin, on s'arrêtera sur des moments de discussion durant lesquels ces derniers réagissent vivement et s'offusquent, alors que de l'autre côté, ils acceptent le plus souvent le bien-fondé des violences qu'ils ont commises. Nous nous concentrons ici sur une thématique d'indignation qui revient dans les deux contextes et concerne le rapport à l'argent et à la gestion des ressources dans l'exercice de leurs fonctions.

Mots-clés : Analyse de témoignages, auteurs de violences de masse, anthropologie des intolérables, justifications, situation d'entretien, Allemagne nazie, dictatures du Plan Condor.

[En] In this paper, we place in perspective testimonies of mass violence perpetrators in cases where the perpetrators come from two very distinct socio-historical contexts, but share the feature of being produced in extrajudicial situations, often in jail, and take the form of conversations with journalists or fellow inmates. Firstly, we will bring to light the power relationships characteristic of these interactions, as well as strategies carried out by the interlocutors to lead these "veterans" beyond their face-work (Goffman, 1967) and, through the framework of the encounter, make them speak more consistently about their violent acts. Secondly, we will deal with how documents of this type can give us a privileged access to questions of morality in situations of mass violence. To this end, we will look closely at places in the discussions where the mass-murderer reacts strongly and feels offended, while on the 
other hand he often asserts the legitimacy of the violence he committed. We will focus here on the thematics of indignation in both contexts and more specifically on the relationships to money and the management of resources that emerge for the perpetrators in the course of their duties.

Keywords: testimonies of perpetrators (of mass violence), justification, interview situation, moral anthropology, Nazi Germany, Last Military Dictatorships in Argentina and Chile.

\section{Introduction}

Cet article interroge et explore des témoignages de participants directs à des violences de masse qui ont été produits postérieurement à la chute du régime dans lequel ils ont sévi. Si ces prises de parole font référence à des configurations sociohistoriques de violences extrêmes et étatiques bien distinctes, elles partagent cette caractéristique de sortir du cadre juridique et de provenir de situations de dialogue. Trois des situations de dialogue étudiées dans cet article se rapportent à l'Allemagne nazie, deux à la dictature de la Junte Militaire en Argentine et une à la dictature de Pinochet au Chili.

Ces documents doivent toujours leur existence à des circonstances assez exceptionnelles. Quand ils ne résultent pas de trop rares entretiens effectués avec un journaliste, ces propos d'anciens auteurs de violences de masse sont collectés à leur insu. On retrouve dans ce cas celui de ces hauts-gradés de la Wehrmacht mis sous écoute alors qu'ils étaient détenus dans des prisons anglaises en 1944 (Neitzel, 2010 ; Neitzel et Welzer, 2013) ou celui du résistant polonais Kazimierz Moczarski qui a restitué de manière scrupuleuse et pénétrante les longs mois qu'il a passés en cellule avec l'officier SS Jürgen Stroop de mars à novembre 1949 (Moczarski, 2011), ces deux écrits faisant partie de nos sources pour cet article. Arrêté par des membres du $\mathrm{MBP}^{1}$, Moczarski est condamné à dix ans de prison pour «intelligence avec l'ennemi fasciste » alors qu'il a, bien au contraire, participé à des actions notoires de résistance contre le nazisme parmi lesquelles des aides logistiques aux insurgés du ghetto de Varsovie (Panné, 2011). Les services secrets le gardent pendant neuf mois dans la même cellule de prison que l'ancien SS Jürgen Stroop pour mieux l'assimiler à ceux qu'il a combattu. En effet, Stroop est précisément celui qui a dirigé les opérations d'anéantissement du ghetto de Varsovie, et est alors condamné à mort. Les deux détenus partagent leur cellule de la rue Rakowiecka avec un second SS du nom de Gustav Schielke. Les écrits de Moczarski donnent des informations

\footnotetext{
${ }^{1}$ MBP : Ministère de la sécurité intérieure, police politique de la République populaire de Pologne entre 1945 et 1954.
} 
fournies sur les attitudes du SS Stroop et sur la dynamique des discussions qu'il a entretenues dans le cadre de ce huis-clos à trois.

En dehors du cas Stroop, les trois autres sources étudiées dans la première partie de cet article sont des ouvrages rédigés par des journalistes et composés d'extraits de leurs entretiens. Il y a la conversation menée sur plusieurs mois en 1971 par la journaliste Gitta Sereny dans une prison de Düsseldorf avec le SS Fritz Stangl, ancien commandant du camp d'extermination de Sobibor et de Treblinka.

Dans le contexte des dictatures militaires du Cône-Sud latino-américain, on se basera sur le long entretien réalisé en 1995 par le journaliste argentin Horacio Verbitsky avec le capitaine à la retraite Adolfo Scilingo. Au cours de cette conversation, ce militaire déclare avoir jeté trente détenus politiques dans le Rio de la Plata depuis un avion après qu'ils aient été drogués. À cette série de dialogues, s'ajoute encore celui produit par la journaliste chilienne Nancy Guzmán dans une prison de haute-sécurité de Santiago avec le tortionnaire Osvaldo Romo qui a sévi dans le centre de détention situé à la Villa Grimaldi entre 1974 et 1976.

\section{En conversant avec les bourreaux : enjeux et étapes de cet article}

Ces entretiens sont la rencontre de deux préoccupations aussi différentes que vives et fortes. En effet, quel ancien auteur de violence se prêterait sans motivation spécifique au jeu qui consiste à revenir sur son passé quand il n'en est en rien contraint? Et quel journaliste mènerait volontairement de tels entretiens sans que le sens de son action ne parte de questionnements très personnels ? Une conversation aussi improbable ne se déroule en aucun cas sans obstacles et la première partie de notre analyse se consacrera à étudier les enjeux symboliques liés à sa dynamique. Nous nous situons dans une perspective de recherche que l'on appelle en sociologie l'interactionnisme symbolique. Mais, suivant les critiques justifiées du caractère déshistoricisant des «sociologies de la vie quotidienne» (Elias, 1995) dont une telle approche fait partie, nous envisagerons les sujets individuels de nos sources comme des produits historiques dont les propos, en plus et au-delà d'être le résultat de l'interaction étudiée, peuvent constituer des renseignements de premier ordre sur des points de vue éprouvés dans la perpétration de violences extrêmes au nom de l'État.

Comme on le mentionne dans le titre de cet article, nous cherchons à exploiter de telles sources dans le but de mieux étudier deux caractéristiques liées aux violences de masse du point de vue de ceux qui les ont commises. Il y a d'une part la question du déni, qu'il soit volontaire ou engendré par des mécanismes dont le locuteur n'a pas pleinement contrôle. Ainsi, seront explorées des possibilités de parvenir à un degré moindre de déni des violences chez l'ancien auteur de violences à travers ce type d'interactions. D'autre part, il y a la question des valeurs morales qui viennent soit accompagner, soit directement justifier la violence extrême, et dont l'existence dans 
ce type de prises de parole mérite qu'on s'y attarde. Ce dernier objet de questionnement et d'étude s'avère essentiel dans la recherche sur les violences, en ce sens que de tels individus ont généralement été envisagés dans leur amoralité alors que le fait qu'ils expriment des valeurs morales devrait nous pousser à poser les choses autrement.

\section{Briser la glace}

Dans cette première partie du développement, nous avons recours à quatre sources d'entretiens (Moczarski, 2011 ; Sereny, 2007 ; Verbitsky, 1995 ; Guzmán, 2000) ${ }^{2}$. Il convient de bien prendre en compte les conditions particulières qui relient ces quatre situations de dialogue. Nous avons affaire à des soldats qui ont désormais perdu grades et galons et se retrouvent, dans trois cas de figure sur quatre, confinés dans un espace carcéral. Dans le cas de Scilingo (Verbitsky, 1995), ses entretiens ont lieu en période d'amnistie, mais les actes qu'il a commis et, par ailleurs, avoués, ont été décrits dans le rapport de la Commission Nationale sur la Disparition des Personnes (CONADEP, 1984) et sont moralement condamnés par le régime démocratique, en place depuis alors onze ans.

On a de fait changé de régime d'historicité ${ }^{3}$ depuis le moment où les violences de masse en question ont été produites. Dès lors, la tension se fait grandement ressentir parce que ceux qui posent les questions auraient, sous le régime des bourreaux, fait partie de la catégorie des victimes de ceux qu'ils questionnent. Du côté des auteurs de violences, les schèmes de rapports de pouvoir semblent disposer d'attaches directes avec l'esprit de l'époque dans laquelle les violences ont été commises. Passé et présent s'entremêlent. Moczarski explique que l'ancien officier SS Stroop souhaite lui laisser sa place dans le seul lit disponible de la cellule et dormir à même le sol avec l'ancien SS Schielke. Ayant perdu la guerre, il considère que Moczarski appartient au «peuple vainqueur, à celui qui gouverne ici, au peuple des seigneurs, par conséquent» (Moczarski, 2011, 42-43). Moczarski précise que Stroop avait alors utilisé l'expression Herrenvolk.

Engager et maintenir la conversation avec un ancien auteur de violences de masse implique une gestion de ces asymétries de relation de pouvoir. On ne retrouve pas un déséquilibre relationnel dans les premiers échanges courtois entre Fritz Stangl,

\footnotetext{
${ }^{2}$ Par la suite, quand nous nous pencherons sur des valeurs morales exprimées par ces anciens auteurs de violences, nous aurons l'occasion d'inclure quelques autres sources de ce type dans l'analyse.

${ }^{3}$ Expression reprise de l'ouvrage suivant: Hartog, F. (2003), Les Régimes d'historicité, Paris : Seuil. Le régime d'historicité correspond ici à la représentation du temps (rapports au passé, au temps présent et au futur) dans une communauté donnée, qui deviendra caduque ou sera bouleversée lors de la fin non-souhaitée du « régime » en question.
} 
l'ancien commandant du camp d'extermination de Treblinka et la journaliste Gitta Sereny. En revanche, il est fortement présent lors de la première visite de la journaliste Nancy Guzman auprès de la cellule pénitentiaire de l'ancien tortionnaire Osvaldo Romo à Santiago du Chili. Le cadre de discussion, non-institutionnalisé et genré, avec une femme qui appartient à la même génération que ses victimes, correspond d'entrée de jeu pour Romo à une bonne occasion de réhabiliter son ancien statut, ce non pas seulement pour se valoriser, mais pour continuer à interagir en terme de terreur. «Ils me détiennent ici parce qu'ils m'ont capturé au Brésil, mais, moi, je reçois des ordres militaires. S'ils me disent de te faire sortir les tripes, je le fais ici et maintenant » (Guzmán 2000, 40-41). En d'autres termes, Romo ne se réfère pas au passé mais en vient à soutenir, de manière invraisemblable, que l'ordre institutionnel dans lequel il a agi a toujours cours. En s'attribuant son statut passé d'agent de la DINA et en le revivant, il emploie de manière brutale une ressource psychologique de prétention à l'omnipotence, qui découle du savoir-faire acquis pendant son travail dans les centres de torture. Pour rééquilibrer les rapports de pouvoir, Nancy Guzmán ne se prend pas au jeu de son interlocuteur et lui rappelle les conditions objectives du cadre de leur interaction, que ce dernier finira par accepter. «Romo, vous ne croyez pas que tout cela est un peu ridicule ? Je suis libre, j'ai ici à mes côtés un gendarme (...) et avant même que vous n'avanciez la main sur moi, ils vous tireront une balle en pleine tête. Quand je sors d'ici, j'irai me promener dans les rues de Santiago pour apprécier la beauté du printemps. Et vous ? Vous serez ici enfermé » (Guzmán, 2000, 40-41).

D'une toute autre manière, le journaliste Horacio Verbitsky doit également rappeler au capitaine vétéran Scilingo, non sans une once d'ironie, les conditions de liberté qui régissent leur interaction dans son salon, au moment où le militaire à la retraite en vient à parler en détail des exactions qui étaient commises dans le centre de détention où il travaillait.

— SCILINGO : Il y a quatre choses qui me rendent très mal à l'aise. Les deux « vols » que j'ai faits, la personne que j'ai vue torturée et le souvenir du bruit des chaînes et des fers qui leur étaient mis au pied. Je les ai vus une fois, mais je ne peux pas oublier ce bruit, je ne veux pas parler de cela. Laissez-moi partir.

- VERBITSKY : Je vous rappelle que l'on n'est pas à l'ESMA ${ }^{5}$, ici. Vous êtes là de par votre volonté et vous pouvez vous en aller quand vous voulez. — SCILINGO : Oui, je sais, ce n'est pas ce que je voulais dire. Il y a des détails qui sont importants, mais cela me fait du mal d'en parler (Verbitsky, 1995, 54).

\footnotetext{
${ }^{4}$ La DINA (Dirección de inteligencia nacional, en français : Direction nationale du renseignement) était la police politique chilienne pendant la dictature militaire d'Augusto Pinochet.

${ }^{5}$ Escuela Superior de Mecánica de la Armada: Il s'agit probablement du centre clandestin de détentions le plus emblématique de la dictature de la junte militaire en Argentine, institution dans laquelle a travaillé Scilingo.
} 
On constate, dans ce cas de figure, que durant toute leur discussion, le journaliste aura l'ascendant sur le militaire. Soumis, ce dernier semble s'être habitué à agir en exécutant et a si bien intériorisé les situations d'enfermement et de pression physique et psychologique dans lesquelles se trouvaient les personnes subissant un interrogatoire à l'ESMA qu'il les transpose involontairement dans cet interview. Par un rappel du cadre de l'interaction et en lui relevant son lapsus, Verbitsky cherche alors à remettre son interlocuteur à sa juste place, de même qu'il l'aidera à se défaire de conceptions idéologiques qui entravent l'avancée de son propos (Payne, 2008, 70).

\section{3. Ébrécher la façade}

En employant l'expression «ébrécher la façade » pour nommer cette section, on fait directement allusion à la notion de «face», développée par le sociologue Erving Goffman en particulier dans la première partie de son ouvrage Rites d'interaction. À travers des postures type et des discours préconstruits, les énonciateurs cherchent à «garder la face », ce qui pour Goffman correspond à donner une cohérence entre l'image que l'on veut donner de soi à son interlocuteur et le récit qui nous représente dans le dialogue (Goffman, 2003, 10). La teneur des propos dépendra largement de ces enjeux symboliques de l'interaction; que l'interrogé cherche à se faire courtois (le S.S. Stangl), qu'il se montre orgueilleux et droit dans ses bottes (le S.S. Stroop) ou encore qu'il dialogue sur un mode équivalent, mais provocant et fruste (le tortionnaire chilien Romo).

Comment contrer cette logique de l'interaction? On peut dire que les quatre questionneurs sont parvenus sur la longueur et au bout d'un grand investissement à faire par moment tomber ces masques. Chacun d'entre eux a fait usage de marges de manœuvre adaptées à sa propre personnalité et ses préoccupations, tout autant qu'en fonction du profil d'interlocuteur qu'ils avaient en face. L'analyse est portée davantage ici sur ce second facteur dans la production de l'interaction, bien que les deux ne soient pas dissociables.

Gitta Sereny met en avant le fait que ses premiers échanges en cellule avec l'ancien commandant de Treblinka se sont déroulés en bonne courtoisie, mais les propos sur son passé qui en sont ressortis ne présentaient pas le moindre intérêt : «Les arguments, la phraséologie, les termes mêmes dont il se servait avaient la résonance agaçante des auditions de tous les procès de criminels nazis. Il n'avait rien à se reprocher : il avait toujours eu des supérieurs hiérarchiques ; il n'avait fait qu'obéir aux ordres ; personnellement il n'avait jamais fait de mal à personne » (Sereny, 2007, 26). Assez rapidement, elle va devoir s'accorder sur la conversation qu'elle cherche à mener avec le bourreau, ou pour le dire autrement, sur le genre de discours qui en découlera. Dépassant le cadre d'une simple interview, elle souhaite que Stangl maintienne un propos de fond sur son parcours biographique. En le faisant plonger par ses questions dans le passé le plus lointain et remonter jusqu'à la guerre, elle s'ef- 
force de le détourner de cette vision très sommaire de son passé qu'il expose au départ et qui, sous la forme du discours de façade semble avoir durci comme une coque dans son esprit.

Quand Sereny en vient à aborder l'époque du camp d'extermination avec Stangl, il arrive que ce dernier parle «en passant»d'une victime avec laquelle il a eu une brève interaction, comme avec cet homme sur la rampe de Treblinka. Dans ces caslà et de manière constante, la journaliste lui pose la question suivante : «Savez-vous ce qui lui est advenu ?». En posant de telles questions, Gitta Sereny participe à une forme de resubjectivation de la victime aux yeux de son bourreau, à une réhumanisation de ceux qu'il mentionne dans le cadre étroit de ce contact et dont le sort funeste est pleinement dénié. À travers ce questionnement qui explicite un manque problématique dans le récit exposé par Stangl, Sereny se situe sur une ligne de faille sur laquelle elle va revenir dès qu'elle en a l'occasion. En effet, ce questionnement et les réponses inadéquates formulées par Stangl qui en découlent, mettent à jour un phénomène de compartimentation ${ }^{6}$ de la pensée qui lui permettait de s'accommoder du génocide qui avait cours devant sa fenêtre et auquel il participait pleinement. On découvrira dans l'entretien que cette compartimentation ne tient plus quand Stangl se retrouve face aux baraques de déshabillage, qui constituent, dit-il, l'endroit qui lui a été le plus insupportable dans le camp de concentration. Elles semblent bien correspondre au lieu concret de franchissement de la barrière mentale qu'il s'était construite, avec d'un côté des personnes bien vivantes (qu'il considérait cependant comme des « cargaisons ») et de l'autre les cadavres. «Je repoussais [les baraques de déshabillage] du plus profond de moi-même. Je ne pouvais pas les affronter ; je ne pouvais pas leur mentir ; j'ai évité par tous les moyens de parler à ceux qui allaient mourir ; je ne pouvais pas le supporter »(Sereny, 2007, 217).

Revenons à présent à la situation d'entretien entre Osvaldo Romo et Nancy Guzman pour voir de plus près les stratégies envisagées par la journaliste pour que cet homme de main du régime de Pinochet s'exprime plus en détail sur les violences extrêmes qu'il a lui-même commises. Face au caractère orgueilleux et agressif de Romo, Nancy Guzman ne peut se placer dans des rapports de force explicites. Elle envisagera des stratégies indirectes. En effet, dès qu'elle utilise des termes disposant d'une connotation accusatrice, ce dernier renforcera son discours de façade, quand bien même celui-ci est particulièrement inconsistant. Il récuse avoir été un tortionnaire et conteste l'usage du mot torture quand elle lui demande s'il a participé à celles-ci. Ainsi, elle s'efforce d'ôter toute valeur accusatrice à ses mots et formulera certaines de ses questions à partir d'un positionnement invraisemblable, mais qui

\footnotetext{
${ }^{6}$ Ce processus de compartimentation de la pensée chez les auteurs de violences de masse est introduit et développé dans les recherches du sociologue néerlandais Abram de Swaan. Un tel mécanisme est notamment exposé dans son excellente allocution d'ouverture au Troisième congrès de l'Association Française de Sociologie, et accessible en ligne (De Swaan, 2009).
} 
semble fonctionner en ce qu'elle se mettra au même niveau d'acceptation des violences que son interlocuteur.

— ROMO : Il y avait l'électricité, le palo de arara comme on dit au Brésil, les sous-marins...

— GUZMAN : Je ne les connais pas. Pouvez-vous m'expliquer en quoi ils consistent ? (...)

- GUZMAN: Pourquoi leur mettiez-vous un bandeau devant les yeux ?

(Guzmán, 2000, 165).

En posant des questions naïves et en jouant sur la non-conscience de l'individu du caractère effroyable des actes qu'il décrit, elle fait largement revenir ce dernier sur ses exactions, sans qu'il s'y confronte pour autant. Romo éprouve un certain besoin de parler de ce qu'il «faisait », de son travail, et cela lui procure de la fierté. Ce ressenti le ramène à l'époque qui apparaît pour lui comme « la plus prestigieuse » de son parcours biographique, celle où il exerçait un pouvoir de vie et de mort sur des centaines de personnes. Et comme l'explique Guzmán autre part dans son propos, et en lien avec les préoccupations qui l'ont poussée à réaliser cet entretien, cette banalisation extrême de la violence chez son interlocuteur est un cas-limite, qui reflète le fait que le Chili de 1995, soit au moment de l'entretien, est un pays encore trop habitué à la torture et loin d'être en mesure de la condamner moralement (Ibid., 17).

\section{Les intolérables chez ces anciens auteurs de violences de masse}

Ces situations de dialogue, notamment quand l'un des interlocuteurs a aussi officié pour le même régime et partage par-là un même espace de préoccupation, mettent en évidence un certain nombre de valeurs morales exprimées. Partant du point de vue que ces personnes acceptent ou ont fini par accepter le bien-fondé des violences qu'elles ont commises, on en vient alors à se demander à quoi ces derniers peuvent encore tenir. Dans cette optique, il s'avère judicieux de revenir sur ces moments de la discussion durant lesquels ces derniers s'offusquent du comportement des leurs ou de certaines accusations que l'on émet à leur encontre. Parmi la pluralité de topoï qui suscite l'indignation des auteurs de violences de masse, dans ces témoignages, on en retrouve un qui concerne le rapport à l'argent et aux ressources. Ainsi, ils trouvent intolérable que certains types de ressources, pécuniaires de surcroît, fassent l'objet d'un usage irraisonné, ou qu'elles puissent venir détourner le sens de leur action. Ceci s'applique tout autant à celle de leurs pairs. La suite de cet article se concentre sur cet ensemble de valeurs, parce qu'elles reviennent assez souvent et ostensiblement dans les propos des auteurs de violences de masse et ce, dans les deux contextes larges de perpétration à l'examen ici.

On les retrouve notamment dans les récits échangés par les soldats hauts-gradés faits prisonniers avant la fin de la guerre et mis sous écoute pendant leur période de 
détention en Angleterre. Le général Kittel de la Wehrmacht explique à ses camarades avoir donné l'ordre d'interrompre une fusillade de Juifs de Dvinsk en présentant son intervention comme suit: «Si vous abattez des gens dans la forêt ou n'importe où pourvu qu'on ne voit rien, c'est votre affaire. Mais, $j$ 'interdis tout simplement que l'on tire ici un jour de plus. Nous prenons notre eau potable dans des puits, nous n'avons plus que de l'eau infestée par les cadavres » (Nietzel et Welzer, 2013, 180).

Aucunement alarmé par les atrocités commises, ce général de la Wehrmacht considère en revanche inacceptable que les membres de la Sicherheitsdienst (service secret de la SS) qu'il côtoyait à ce moment-là, puissent à ce point faire défaut en termes de bon sens logistique et de savoir-faire fondamental d'hygiène collective. De telles méprises choquent le narrateur, en même temps qu'elles donnent à son propos ce caractère «haletant» qui rend son histoire digne d'être racontée à ses compagnons de cellule. Comme le commente Harald Welzer dans l'introduction à son livre Soldaten, ces propos produits entre soldats de compagnies différentes, expatriés et emprisonnés ont pour fonction caractéristique de «susciter la tension et l'intérêt entre pairs, tout en laissant la place et la possibilité que s'y ajoutent les commentaires et les histoires des autres. (...) Les gens ne s'entretiennent pas seulement pour échanger des informations, mais aussi pour construire une relation, tisser une complicité et s'assurer qu'ils font bien partie d'un seul et même monde. Ce monde est la guerre et c'est ce qui rend ces conversations très inhabituelles, mais ce seulement pour la lectrice ou le lecteur d'aujourd'hui, et pas pour les soldats » (Welzer, 2011, 11) ${ }^{7}$.

Dans l'autre huis-clos impliquant Kazimierz Moczarski, le SS Stroop et le SS Schielke, c'est sous la forme de virulentes controverses entre les deux anciens nazis que viennent s'esquisser quelques frontières de ce qui apparaît pour Stroop comme moralement intolérable (Bourdelais et Fassin, 2005). Ainsi, d'après Moczarski, Stroop s'est senti très offensé et n'a plus adressé la parole à Schielke pendant trois jours, après que ce dernier ait critiqué le gaspillage de ressources financières occasionnées par les cérémonies «pseudo-antiques » de hauts-gradés auxquelles il était pleinement intégré (Moczarski, 2011, 153).

De même, alors qu'il décrit avec le plus grand mépris le SS Oberführer qui l'a précédé dans les tentatives d'annihilation du ghetto de Varsovie, Stroop se crispe lorsqu'on accuse ce membre de sa corporation d'avoir été corrompu. "C'est un mensonge! s'écria Stroop. Les officiers SS n'acceptaient pas les pots-de-vin!» (Ibid.,

\footnotetext{
${ }^{7}$ Considérant que dans ce passage cité de Welzer (Nietzel \& Welzer, 2013, 15), la traduction française ne met pas assez en évidence les fonctions proprement communicationnelles de ces discussions entre soldats que suggère l'auteur pour pouvoir en comprendre la structure et la raison d'être, nous nous sommes permis de fournir ici une traduction personnelle plus proche de la version allemande.
} 
262). À la suite de la réaction très vive et du déni qu'elle implique, Stroop finira par intégrer à l'intérieur de son récit de la destruction du ghetto de Varsovie la présence d'une corruption de certains officiers SS, et en particulier du prédécesseur à son poste. Mais il la présente comme étant le produit de l'attitude insidieuse et active d'autres corporations que la sienne, et surtout des Juifs : «Többens [le propriétaire des usines de travaux forcés du ghetto de Varsovie] (...) et les directeurs et propriétaires juifs de son consortium l'engraissaient certainement. Ils lui offraient du cognac et expédiaient de la mangeaille à sa famille dans le Reich ${ }^{8}$ (Ibid., 267). De tels principes de parcimonie ou d'incorruptibilité sont pourtant loin d'être respectés dans les faits par ces auteurs de violences, dans leur contexte d'action. Mais Stroop veut y croire et tient assez viscéralement à ce que sa corporation soit présentée sous un tel jour. Cet écart vient montrer à quel point des valeurs fantasmées ne sont pas uniquement attribuées à l'ennemi, mais aussi à la corporation d'appartenance.

Parmi les usages de l'argent qui apparaissent comme particulièrement insupportables, on retrouve également l'attitude qui consiste à faire du profit sur les valeurs que l'on défend, alors que celles-ci devraient sublimer toute recherche de gain individuel. Quand Stroop revient sur son enfance, il s'indigne tout particulièrement de ce que le prince de sa région, la Lippe, ose prélever de l'argent auprès des visiteurs de la statue géante d'Arminius dans la forêt de Teutobourg : un monument clé dans la formation de sa propre ferveur nationaliste. «Dites-moi vous-mêmes Herr Moczarski, si vous trouvez digne d'un patriote de tirer profit de ceux qui fortifient le sentiment national en se rendant au monument du grand Germain? » (Moczarski, 2011, $65)$.

Dans le contexte argentin, la mise en avant du «travail bien fait » sans que celui-ci ne soit détourné à des fins personnelles, contrairement à ses ennemis, apparaît dans les propos d'un agent au service de la dictature argentine comme Astíz. Dans un entretien datant de 1998, il déclare qu'en rendant possible l'enlèvement et l'assassinat des trois membres fondatrices des Mères de la Place de Mai en décembre 1977, il avait « accompli son travail » et n'a en cela rien à se reprocher. Puis il exprime juste après et comme par contraste être dérangé par le fait que ces femmes exploiteraient aujourd'hui leur statut de mères de disparus «à des fins de commerce, d'argent ou de politique » (Cerutti, 1998, 10).

\footnotetext{
${ }^{7}$ du livre «Anständig geblieben. Nationalsozialistische Moral » de Raphael Gross (2009) qui traite de Konrad Morgen, un juge SS anti-corrpution à Auschwitz. Voir aussi les ouvrages suivants : Longerich, P. (2010), Himmler, Paris : Éd. Héloïse d'Ormesson et Ingrao, C. (2010) Croire et détruire. Les intellectuels dans la machine de guerre SS, Paris : Fayard.

${ }^{8}$ Sur cette question de l'interdit moral de la corruption chez les SS, on pourra se référer au chapitre
} 


\section{Autour du statut des valeurs morales repérées}

S'il y a lieu de s'intéresser au contenu des conceptions de ces anciens auteurs de violences extrêmes qui émergent à des moments privilégiés de l'interaction, il est également souhaitable de mieux saisir le type de justifications morales auxquelles on est confronté. Dans ce but, et toujours à partir d'exemples qui proviennent de la même thématique large de valeurs exprimées, nous proposons trois catégories d'analyse qui viendraient les distinguer.

Dans l'état actuel d'étude de telles sources, nous considérons en effet que les justifications morales énoncées varient en nature, à savoir qu'elles disposent d'une origine, d'un mode d'appropriation par l'acteur social et sont accompagnées d'un niveau de conviction bien distincts, en fonction de si ces valeurs se sont formées avant, pendant les violences ou encore après que le régime durant lequel ils ont sévi soit tombé.

Or, dans l'étude de ces dialogues en tant que récits de vie fragmentaires et sélectifs, il apparaît possible d'évaluer le moment de formation des valeurs chez l'auteur de violences en portant l'attention non plus au temps de l'énonciation, qui a été surtout examiné jusqu'à présent dans l'article, mais au temps raconté (Ricoeur, 1986). En l'occurrence, ces valeurs morales qui accompagnent les réactions vives de l'auteur de violence, loin d'être de simples « rationalisations rétrospectives » et immédiates sur des actions passées, sont dans bien des cas fortement rattachées au moment de controverse ou à la situation critique évoquée dans le récit et au travers desquels elles se sont formées en lui. Et de fait, ces situations critiques ou controverses se situent soit pendant la pratique des violences, soit à une période antérieure ou ultérieure à celle-ci dans la trajectoire de vie du «bourreau ».

On trouve donc d'abord des valeurs exprimées pendant l'entretien que l'on peut situer dans le temps raconté en amont ou bien en marge des violences commises. Les controverses sur des usages transgressifs de l'argent qui font réagir vertement Jürgen Stroop en constituent un exemple. Celle qui est liée à sa région d'origine - le fait que les visiteurs allemands doivent payer pour visiter la statue d'Arminius - fait intrinsèquement partie de conceptions que Stroop a développées dans son milieu culturel, durant sa jeunesse et qui se sont avérées compatibles avec l'idéologie nationalsocialiste. Le caractère inavouable de l'usage dispendieux des finances par les élites du parti au travers de leurs cérémonies «pseudo-antiques » est contemporain de sa montée en grade dans les instances du parti. Renforcées pendant sa longue carrière dans la S.S., de telles valeurs prennent alors un tour corporatiste où elles participent à la sauvegarde de leur image, quand bien même les pratiques de corruption et de gaspillage critiquées avaient manifestement souvent cours.

Dans un second temps, des valeurs apparaîtront dans leurs propos comme ayant été forgées dans la période de violences de masse et spécifiquement liées à de telles situations, vécues et commises. Elles constituent bien souvent un enjeu de rehiérar- 
chisation morale, entre accommodation à la violence extrême et duperie de soi, que l'on pourra notamment retrouver dans les assertions du commandant du camp de Treblinka lorsqu'il déclare à la journaliste Sereny qu'il ne tolérait pas que les membres de son personnel puissent profiter des biens des victimes.

— SERENY : Est-ce qu'il vous est arrivé de parler à des gens qui arrivaient [sur la rampe de Treblinka] ?

- STANGL: Parler ? Non. Mais je me souviens qu'une fois - ils étaient tous là, à peine débarqués - un Juif s'est approché de moi en disant qu'il avait une réclamation à faire. (...)

Un des gardes lituaniens lui avait promis de l'eau s'il lui donnait sa montre. Il avait pris la montre mais n'avait pas donné d'eau. Bon, ce n'était pas correct n'estce pas ? De toute façon je n'admettais pas le chapardage. (...) Je les ai fait aligner et retourner les poches.

- SERENY : Face aux prisonniers?

- STANGL: Oui, pourquoi pas? Quand il y a réclamation, il faut mener l'enquête. Bien entendu, nous n'avons pas retrouvé la montre - celui qui l'avait s'en était débarrassé.

- SERENY : Qu'est-il advenu du plaignant ? (Sereny, 2007, 181)

Dans cette situation d'interaction, Stangl vante une prise de décision individuelle au travers de laquelle il aurait à ses yeux affirmé un principe de justice et de moralité, tout en excluant de sa pensée, bien entendu, que les mises à mort massives étaient le cadre et la raison d'être de telles situations. Ces valeurs de probité constituent des duperies de soi qui amoindrissent son sentiment de responsabilité dans le processus d'extermination. D'autre part, nous avons affaire dans ce cas de figure à l'évocation d'un souvenir-écran (Freud, 1990), comme il arrive souvent d'en trouver dans ce genre de témoignages, soit le souvenir d'une situation réelle mais extrêmement peu représentative qui vient se superposer et effacer les atrocités commises au quotidien.

Enfin, nous pouvons aussi être confrontés à l'évocation de principes moraux qui sont envisagés par l'auteur de violences en aval de celles-ci, et après la chute du régime. Ces justifications morales constituent moins des moyens de s'accommoder des violences commises qu'elles ne participent à une ligne de défense produite a posteriori, en concertation ou non avec d'autres anciens membres du régime.

On en trouvera un bon exemple dans un passage des mémoires du tortionnaire argentin Vergéz, qui pendant une longue page se défend d'avoir fait du recel avec les biens de personnes qu'il a fait séquestrer (Vergéz, 1995, 248-249). Ce passage se situe dans son récit à une période de procès qui suit assez directement la fin de la dictature et fait référence à une accusation émise à son encontre. En d'autres termes, on peut situer la formation d'un tel discours dans l'esprit du locuteur avec toutes les prétentions morales qui l'accompagnent à ce moment - critique pour lui - de l'après-dictature, quand bien même il se réfère à son action de l'époque des violences. 
Cette façon de se présenter dans ses mémoires, qui ont été écrits à l'attention d'un lectorat acquis à sa cause, comme une personne qui ne faisait pas du profit illicite dans son travail, constitue une manière très peu convaincante de détourner l'attention du public des violations des droits de l'homme qu'il commettait au même moment. Il s'agit, comme souvent dans les justifications morales de ce troisième type formées dans l'après-dictature, de déclarations énoncées avec une particulière mauvaise foi, puisque comme le rappelle le journal Página 12 dans un article datant de $2000^{9}$, Vergéz n'est pas seulement allé jusqu'à voler les meubles, la vaisselle, les habits de l'une de ses victimes avec son contingent des forces armées, mais il a également réalisé un chantage auprès de la famille de celle-ci pour qu'elle lui remette une forte somme d'argent contre sa libération.

\section{Conclusion}

Nous avons souhaité dans cet article donner à voir le caractère heuristique de ces dialogues hors-procès, dès lors que l'on oriente son intérêt de recherche sur la subjectivité dans la violence commise ou alors sur la présence d'un ordre moral qui accompagne ces discours sur la transgression en puissance de l'interdit du meurtre.

Dans sa confrontation avec l'auteur de violences de masse d'un régime précédent, le journaliste aura déployé des stratégies qui ont été mises ici en avant et lui permettent de déjouer ou rééquilibrer les contraintes de rapport de force qui se présentent dès le début de ces conversations, pour le moins atypiques. Dans cette élaboration à la fois maïeutique et éminemment conflictuelle du souvenir du bourreau, on se sera penché sur les passages où les énonciateurs reviennent sur des fragments traumatiques de leur passé qu'ils n'auraient pas envisagé d'aborder, parfois en butant sur leurs paroles. Ces moments privilégiés importent pour ce qu'ils disent sur les mécanismes qui auront permis au bourreau de dénier sa violence et de la rendre par-là aussi supportable que possible. Mais aussi, ils sont intéressants pour apprécier l'entrée de l'interlocuteur dans la pensée du bourreau ou sa logique de pensée, qui constitue le moyen d'accéder à ces retours sur les violences commises.

D'autre part, en poussant l'auteur de violences dans ses retranchements sans pour autant que l'interaction soit interrompue, il arrive que le codétenu ou le journaliste provoquent la réminiscence de controverses ou de situations critiques de son passé, à partir desquelles s'expriment une réaction d'offuscation ou des valeurs à caractère moral que ce dernier vient à défendre.

Ces moments très spécifiques du dialogue vont occuper toute notre attention durant la seconde partie de cet article, dans une analyse qui, à l'opposé de la première,

\footnotetext{
${ }^{9}$ Página 12, «El capitán Vergez, actual usurero, pierde el pelo pero no las mañas. », Buenos Aires, 31.08.2000.
} 
donne toute son ampleur au poids du temps raconté sur le temps d'énonciation et d'interaction. Ces justifications à dimension morale sont en effet souvent peu dissociables des moments de controverse auxquelles elles se réfèrent dans le récit et au travers desquelles elles ont été forgées. Prenant comme critère d'analyse cette temporalité de référence, nous considérons que ces valeurs morales exprimées se différencient en type, en fonction de si elles se raccordent à la période qui précède les violences, à celle de la pratique des violences de masse ou à l'après-régime.

Dans le premier cas, elles prennent la forme de conceptions dont l'individu en question se sera imprégné au travers de son milieu culturel de provenance et de socialisation. Les valeurs qui sont en revanche directement rattachées dans le récit à des situations critiques dans la pratique de violences de masse s'articulent, elles, étroitement à des mécanismes de déni et d'accommodation qui auront permis à ces personnes de continuer routinièrement leur processus de mise à mort. Enfin, les valeurs morales exprimées liées à des controverses de l'après-dictature auront des traits de discours de façade qui apparaissent autant pour se sauver la face que pour se construire une ligne de défense dans un nouveau régime qui aura pleinement contesté la légitimité de leurs actes.

\section{Bibliographie}

\section{Sources primaires :}

Cerutti, G. (1998). «El asesino esta entre nosotros», Buenos Aires : revista Trespuntos, janvier 1998, pp.7-12.

GuZman, N. (2000). Romo, Confesiones de un Torturador, Buenos Aires : Editorial Planeta.

MoCZARSKI, K. (2011 [1977]). Entretiens avec le bourreau, Paris : NRF Gallimard.

SERENy, G. (2007 [1974]). Au fond des ténèbres. De l'euthanasie à l'assassinat de masse : un examen de conscience, Paris : Denoël.

VERBITSKY, H. (1995). El Vuelo, Buenos Aires : Planeta.

\section{Sources secondaires :}

Bourdelais, P. et FASSIN, D. (2005). Les constructions de l'intolérable : Etudes d'anthropologie et d'histoire sur les frontières de l'espace moral, Paris: La Découverte.

DE SWAAN, A. (2009). «La régression au service de l'État: réflexions sur la violence de masse », La Vie des idées, 29 septembre 2009. URL: http://www.laviedesidees.fr/La-regression-au-service-de-1-Etat.html 
Elias, N. (1995). «Sur le concept de vie quotidienne », Cahiers internationaux de sociologie, 99, pp.237-246.

FREUD, S. (1990 [1899]). «Sur les souvenirs-écrans », in Névrose, psychose et perversion, Paris : PUF, pp.113-132.

GOFFMAN, E. (2008 [1967|). Les rites d'interaction, traduit de l'anglais par Kihm Alain. Paris : Éd. de Minuit.

Gross, R., (2009). Anständig geblieben. Nationalsozialistische Moral, Francfort: Fischer Verlag.

Mosse, G. (1999 [1996]). L'image de l'homme. L'invention de la virilité moderne, Paris : Agora Pocket.

NeITZEL, S. et H. Welzer (2013). Soldats. Combattre, tuer, mourir: Procèsverbaux de récits de soldats allemands, Paris : NRF Gallimard.

Neitzel, S. et H. Welzer (2011). Soldaten. Protokolle vom Kämpfen, Töten und Sterben, Francfort : Fischer.

PANNÉ, J.-L., (2011). «Moczarski : entretien avec un bourreau le SS Jürgen Stroop », revue Arkheia (section critiques de livres).

PAYNe, L. (2008). Unsettling Accounts. Neither Truth Nor Reconciliation in Confessions of State Violence, Duke University Press.

RICOEUR, P. (1986), Du texte à l'action. Essais d'herméneutique II, Paris : Seuil.

WINKIN, Y. (1988), Erving Goffman. Les moments et leurs hommes, Paris : Seuil. 